TRANSACTIONS OF THE

AMERICAN MATHEMATICAL SOCIETY

Volume 354, Number 5, Pages 1997-2012

S 0002-9947(02)02918-5

Article electronically published on January 7, 2002

\title{
TRUDINGER INEQUALITIES WITHOUT DERIVATIVES
}

\author{
PAUL MACMANUS AND CARLOS PÉREZ
}

\begin{abstract}
We prove that the Trudinger inequality holds on connected homogeneous spaces for functions satisfying a very weak type of Poincaré inequality. We also illustrate the connection between this result and the John-Nirenberg theorem for BMO.
\end{abstract}

\section{INTRODUCTION}

The Sobolev embedding theorem says that functions in $W_{\text {loc }}^{1, q}\left(\mathbb{R}^{n}\right)$ actually lie in $L_{\mathrm{loc}}^{r}$ for $r=n q /(n-q)$ when $1 \leq q<n$. A more precise version of this statement is the following local inequality:

$$
\left(\frac{1}{|B|} \int_{B}\left|f-f_{B}\right|^{r}\right)^{\frac{1}{r}} \leq C r(B)\left(\frac{1}{|B|} \int_{B}|\nabla f|^{q}\right)^{\frac{1}{q}}
$$

for any ball $B$. When $q$ tends to $n$ the constant $C$ on the right-hand side blows up, and so the limiting case with $q=n$, i.e., $W_{\text {loc }}^{1, n} \subseteq L_{\text {loc }}^{\infty}$, is false. The correct result in this instance is that $W_{\text {loc }}^{1, n}$ lies locally in the class $\exp L^{n^{\prime}}$, where $n^{\prime}$ is the conjugate exponent to $n$. The corresponding inequality, called Trudinger's inequality, is

$$
\left\|f-f_{B}\right\|_{\exp L^{n^{\prime}}(B)} \leq C\left(\int_{B}|\nabla f|^{n}\right)^{\frac{1}{n}} .
$$

We should mention that it was also derived by Yudovich in [Y]. See [GT], for instance, for a proof in the case that $f$ has compact support. The norm on the left-hand side is the Luxemburg (or Orlicz) norm associated to the function $\Phi(t)=$ $\exp t^{n^{\prime}}-1$ (see Section 2). Trudinger's inequality has proved to be one of the key results in the study of parabolic and elliptic equations at the critical index (which is $n$ in the case of $\mathbb{R}^{n}$ ). In addition, the sharp value for the constant $C$ appearing on the right-hand side (see Moser [Mo], and Adams [Ad] for the higher dimensional version) plays a major role in geometry, especially in the problem of prescribing Gaussian curvatures on spheres.

The papers [HaK1], HaK2], [FPW], and [MP] show how the inequality (11) is really a consequence of the self-improving nature of simpler inequalities, such as (3) and (4) below, and that this self-improving phenomenon holds in great generality.

Received by the editors March 23, 1999 and, in revised form, July 30, 1999.

2000 Mathematics Subject Classification. Primary 46E35; Secondary 46E30, $42 \mathrm{~B} 25$.

Supported by grant ERBFMBICT960939 of the TMR programme of the European Union. This research was carried out during a stay at the Universidad Autónoma de Madrid, and the author wishes to extend his thanks to the Department of Mathematics there.

Research partially supported by DGESIC grant PB98-0106, Spain. 
The following (Poincaré) inequality holds for any $f \in W_{\mathrm{loc}}^{1,1}\left(\mathbb{R}^{n}\right)$ :

$$
\frac{1}{|B|} \int_{B}\left|f-f_{B}\right| \leq C \frac{r(B)}{|B|} \int_{B}|\nabla f| .
$$

This can be deduced from the representation formula $\left|f-f_{B}\right| \leq C I_{1}\left(|\nabla f| \chi_{B}\right)$, where $I_{1}$ is the fractional integral of order $n-1$. An immediate consequence is that

$$
\frac{1}{|B|} \int_{B}\left|f-f_{B}\right| \leq C r(B)\left(\frac{1}{|B|} \int_{B}|\nabla f|^{q}\right)^{\frac{1}{q}}
$$

When $q=n$, this transforms into

$$
\frac{1}{|B|} \int_{B}\left|f-f_{B}\right| \leq C\left(\int_{B}|\nabla f|^{n}\right)^{\frac{1}{n}}
$$

Hajłasz and Koskela HaK2 showed that if $X$ is a connected metric space and if $f \in L_{\text {loc }}^{1}(X)$ satisfies the inequality (5) for all balls $B$ but with $|\nabla f|$ on the right replaced by any function $g \in L_{\text {loc }}^{n}$, then $f$ satisfies (2) (with $|\nabla f|$ replaced by $g$ ). Their proof follows the classical path of showing that $f$ satisfies (1) for all $1 \leq r<\infty$ and that the constant $C$ in (10) is comparable to $r^{1-1 / n}$ as $r \rightarrow \infty$. Expanding the exponential in a power series and using these estimates to bound the sum of the series yields (2).

In this paper we extend the result of Hajłasz and Koskela by showing that the inequality (5) still self-improves to a Trudinger-type inequality when the right-hand side of (5) is replaced by a general functional $a$ that satisfies the condition $T_{p}$ defined below, and that we can insert a very general weight in the inequality. Furthermore, our proof is direct in that it does not depend on passing through the inequalities (11). We also illustrate the connection between this result and the archetypical selfimproving theorem, the John-Nirenberg theorem, by showing that the latter can be viewed as a limiting case of the former obtained by letting $p \rightarrow \infty$ in the condition $T_{p}$.

Let $(\mathcal{S}, d, \mu)$ be a space of homogeneous type, and $a: \mathcal{B} \rightarrow[0, \infty)$ a functional defined on the family $\mathcal{B}$ of all balls in $\mathcal{S}$. Recall that $d$ denotes a pseudo-metric on $\mathcal{S}$ and that $\mu$ is a measure that is doubling with respect to $d$. $K$ will denote the pseudo-metric constant, this constant being 1 in the metric case.

Definition 1.1. Let $1<p<\infty$. We say that the functional $a$ satisfies the $T_{p}$ condition if there exists a finite constant $c$ such that for each ball $B$

$$
\sum_{j} a\left(B_{j}\right)^{p} \leq c^{p} a(B)^{p}
$$

whenever $\left\{B_{j}\right\}$ is a family of pairwise disjoint sub-balls of $B$.

We will use $\|a\|$ to denote the smallest constant $c$ for which (6) holds. We always have $\|a\| \geq 1$. Examples are $a(B)=\left(\int_{B} g^{p}\right)^{\frac{1}{p}}$, where $g \in L_{\text {loc }}^{p}(\mathcal{S})$, and, more generally,

$$
a(B)=\nu(B)^{\frac{1}{p}},
$$

where $\nu$ is a locally finite measure. Observe that these conditions are increasing, in the sense that if $r<s$, then any functional satisfying $T_{r}$ also satisfies $T_{s}$. Also observe that this condition is much stronger than the $D_{r}$ condition considered 
in $[\mathrm{FPW}]$ and $\left[\mathrm{MP}\right.$ to deduce $L^{p}$ type self-improving properties. This condition requires that

$$
\sum_{j} a\left(B_{j}\right)^{r} \mu\left(B_{j}\right) \leq c^{p} a(B)^{p} \mu\left(B_{j}\right)
$$

whenever $\left\{B_{j}\right\}$ is a family of pairwise disjoint sub-balls of $B$.

Theorem 1.2. Assume that $\mathcal{S}$ is connected, that the functional a satisfies the $T_{p}$ condition for some $1<p<\infty$, and that $w$ is a doubling measure on $\mathcal{S}$ that is absolutely continuous with respect to $\mu$. Let $\delta>0$ be given. For any ball $B$ define $\widehat{B}$ to be $(1+\delta) K B$.

If $f$ is a locally integrable function for which there exist constants $\tau \geq 1$ and $\|f\|_{a}>0$ such that for all balls $B$

$$
\frac{1}{\mu(B)} \int_{B}\left|f-f_{B}\right| d \mu \leq\|f\|_{a} a(\tau B)
$$

then there exists a constant $C$ independent of $f$ such that

$$
\left\|f-f_{B}\right\|_{\exp L^{p^{\prime}(B, w)}} \leq C\|f\|_{a} a(\tau \widehat{B})
$$

for any ball $B$.

Note the important fact that the functional $a$ need not depend on $f$, in contrast to the classical case where the functional is given by $a(B)=\left(\int_{B}|\nabla f|^{n}\right)^{\frac{1}{n}}$. In particular, no differentiability structure is needed.

In the proof of the theorem one cannot take $\delta=0$ in general, as we usually do not have enough geometric control to work inside the original ball all the time. For the same reason, when one proves an inequality such as (7) the constant $\tau$ is typically greater than one. If $\mathcal{S}$ has more geometric structure, then there is an attractive, and by now standard, procedure for showing that the constant $\tau$ in a Poincaré inequality such as (7) can be replaced by 1 . It is sufficient that all balls in $\mathcal{S}$ satisfy a chain condition such as the Boman chain condition. This holds, for example, when $\mathcal{S}$ is a length space, i.e., the quasimetric is a metric and the distance between two points is given by the infimum of the lengths of all rectifiable curves joining them. See [B], [Bo], [FGW], [FPW], [GN], [HaK2], [Je], [L2], and the references therein.

The hypothesis that $\mathcal{S}$ be connected can be relaxed. What is really required is Proposition 2.4 below hold. If, for example, $\mathcal{S}$ is connected and a new homogenous space $\mathcal{S}^{\prime}$ is obtained by removing a set of measure zero from $\mathcal{S}$ (and keeping the same quasimetric and measure), then $\mathcal{S}^{\prime}$ satisfies the proposition, and so the theorem is valid for this new space.

The theorem covers a wide variety of previously known special cases. A basic example is the space $\left(\mathbb{R}^{n}, \rho, d x\right)$ where $\rho$ is the metric associated to a collection $X_{1}, \cdots, X_{m}$ of Hörmander vector fields (cf. [FP], [NSW] and [SCa]). The starting point here is the following estimate from [Je]:

$$
\frac{1}{|B|} \int_{B}\left|f(x)-f_{B}\right| d x \leq C \frac{r(B)}{|B|} \int_{B}|X f(x)| d x,
$$

where $|X f|=\sum\left|X_{i} f\right|$. Consequently,

$$
\left\|f-f_{B}\right\|_{\exp L^{Q^{\prime}(B, w)}} \leq C r(B)\left(\int_{\widehat{B}}|X f(x)|^{Q} d x\right)^{1 / Q},
$$


where $Q$ is the "homogeneous" dimension and $1 / Q+1 / Q^{\prime}=1$. This type of estimate was first considered in $[\mathrm{Fr}]$ in the compactly supported case, and the general case was studied [GN] and [L1].

Another example is Buser's version $[\mathrm{Bu}]$ of the Poincaré inequality for complete manifolds $(M, g)$ with Ricci curvature bounded below by $-a^{2} g$ with $a \geq 0$. Here the gradient and measure are the Riemannian ones, and the relevant Poincaré inequality is

$$
\frac{1}{|B|} \int_{B}\left|f-f_{B}\right| \leq C e^{a C r(B)} \frac{r(B)}{|B|} \int_{B}|\nabla f| .
$$

The corresponding Trudinger estimate is

$$
\left\|f-f_{B}\right\|_{\exp L^{n^{\prime}}(B, w)} \leq C r(B) e^{a C r(B)}\left(\int_{\widehat{B}}|\nabla f|^{n}\right)^{1 / n},
$$

The measures on these manifolds need not be globally doubling. Nevertheless, they will be locally doubling. Indeed, on a ball of radius $R$ the measure will satisfy (16) with $c_{\mu}=C e^{a \sqrt{n-1} R}$ and $D$ being the dimension of the manifold (see [SCo]). This local estimate for the doubling constant is all that is needed for the proof of Theorem 1.2 to yield (12).

We will show in Section 4 that Theorem 1.2 is false in general when the hypothesis that $\mathcal{S}$ is connected is dropped. The best estimate that can be obtained in the nonconnected case is a BMO (or exponential) type estimate. In fact, this estimate can be obtained for functionals $a$ satisfying a condition that is considerably weaker than any $T_{p}$, although we need to assume more about $w$.

Definition 1.3. We say that $a \in T_{\infty}$ if there exists a finite constant $c$ such that for each ball $B$ and each ball $B^{\prime} \subset B$

$$
a\left(B^{\prime}\right) \leq c a(B) .
$$

Clearly, $T_{p} \Rightarrow T_{\infty}$ when $p<\infty$. As before, $\|a\|$ will denote the smallest constant $c$ for which (13) holds. The condition $T_{\infty}$ is simply the limit as $p \rightarrow \infty$ of the condition $T_{p}$. Observe that the functional $a(B) \equiv 1$, the functional associated to the space BMO, satisfies $T_{\infty}$. The correct theorem now is:

Theorem 1.4. Suppose that in the previous theorem we remove the hypothesis that $\mathcal{S}$ is connected and instead assume that $a \in T_{\infty}$ and that $w$ is an $A_{\infty}$ weight with respect to $\mu$. Then there exists a constant $C$ independent of $f$ such that for any ball $B$ we have

$$
\left\|f-f_{B}\right\|_{\exp L(B, w)} \leq C\|f\|_{a} a(\tau \widehat{B}) .
$$

This is not much more than the John-Nirenberg theorem for spaces of homogeneous type. The John-Nirenberg result is well known in this context, although the only proof we have seen is that in [ST], and it contains a variety of minor errors. In order to tailor the result to our setting, we give a complete proof of Theorem 1.4 in Section 5. The proof is different than that in [ST].

We would like to thank R. Wheeden for suggesting a technique of Hedberg as an approach to proving Theorem 1.2 (see the last part of Section 3). We are also grateful to P. Hajłasz and P. Koskela for showing us their unpublished work. 


\section{Preliminaries}

A Young function $\Phi$ is a convex homeomorphism from $[0, \infty)$ onto $[0, \infty)$. For any such function we define the norm

$$
\|g\|_{\Phi(L)(B, \mu)}=\inf \left\{\lambda>0: \frac{1}{|B|} \int_{B} \Phi\left(\frac{|g|}{\lambda}\right) d \mu \leq 1\right\} .
$$

Such a norm is called a Luxemburg, or Orlicz, norm. For notational convenience we denote the norm associated to the Young function $\Phi(t)=\exp t^{r}-1$ by $\|\cdot\|_{\exp L^{r}}$.

A quasimetric $d$ on a set $\mathcal{S}$ is a function $d: \mathcal{S} \times \mathcal{S} \rightarrow[0, \infty)$ satisfying

(i) $d(x, y)=0$ if and only if $x=y$,

(ii) $d(x, y)=d(y, x)$ for all $x, y$, and

(iii) there exists a finite constant $K \geq 1$ such that

$$
d(x, y) \leq K(d(x, z)+d(z, y))
$$

for all $x, y, z$.

Given $x \in \mathcal{S}$ and $r>0$, we let $B(x, r)=\{y \in \mathcal{S}: d(x, y)<r\}$ and refer to $B(x, r)$ as the ball with center $x$ and radius $r$. We equip $\mathcal{S}$ with a topology by saying that a subset $U$ of $\mathcal{S}$ is open if for any $x \in U$ there is some $r>0$ for which $B(x, r) \subset U$. Note that the balls defined above are not necessarily open sets in this topology.

A space of homogeneous type $(\mathcal{S}, d, \mu)$ is a set $\mathcal{S}$ together with a quasimetric $d$ and a nonnegative Borel measure $\mu$ on $\mathcal{S}$ such that every ball is a Borel set of finite measure, and the doubling condition

$$
0<\mu(B(x, 2 r)) \leq C \mu(B(x, r))
$$

holds for all $x \in \mathcal{S}$ and $r>0$. Although balls are not necessarily open sets, a result of Macías and Segovia MS says that one can always find a continuous quasimetric $d^{\prime}$ which is equivalent to $d$ (i.e., there are constants $c_{1}$ and $c_{2}$ such that $c_{1} d^{\prime}(x, y) \leq d(x, y) \leq c_{2} d^{\prime}(x, y)$ for all $\left.x, y \in \mathcal{S}\right)$; in particular, every ball is open in the homogeneous space $\left(\mathcal{S}, d^{\prime}, \mu\right)$.

If a ball $B$ is given, then $x_{B}$ denotes the center of $B$, while the radius will be denoted either by $r_{B}$ or by $r(B)$.

We denote by $D=\log _{2} C$ the doubling order of $\mu$, where $C$ is the smallest constant in (15). By iterating (15), we then have

$$
\frac{\mu(B)}{\mu(P)} \leq c_{\mu}\left(\frac{r(B)}{r(P)}\right)^{D}
$$

for every pair $P, B$ of balls such that $P \subset B$.

All constants in the remainder of this section will depend only on those of $\mathcal{S}$, i.e., $K, c_{\mu}$, and $D$.

2.1. A decay estimate. In general there is no lower bound corresponding to (16). However, such a lower bound can be obtained when $\mathcal{S}$ is connected.

Proposition 2.1. If $\mathcal{S}$ is connected, there is a positive constant $\alpha$ for which

$$
c\left(\frac{r(B)}{r(P)}\right)^{\alpha} \leq \frac{\mu(B)}{\mu(P)}
$$

for arbitrary balls $B$ and $P$ such that $P \subset B$ and $B \neq \mathcal{S}$. 
Proof. Let $B=B(x, R)$ be any ball that is not all of $\mathcal{S}$. The sets $\{y: d(y, x)>$ $R /(2 K)\}$ and $\{y: d(y, x)<R /(2 K)\}$ are disjoint, open, and non-empty. The connectedness of $\mathcal{S}$ therefore implies that there is a point $z$ for which $d(z, x)=R /(2 K)$. A simple calculation using the quasimetric shows that $B_{z}=B\left(z, R /\left(4 K^{2}\right)\right)$ lies in $B$ but does not intersect $\lambda B$, where $\lambda=\left(4 K^{2}\right)^{-1}$. The bound (15) implies that $\mu\left(B_{z}\right) \geq \epsilon \mu(B)$ for some positive constant $\epsilon$ depending only on the constants of $\mathcal{S}$. Consequently, $\mu(\lambda B) \leq(1-\epsilon) \mu(B)$. Iteration of this inequality shows that the required inequality holds when $B$ is of the form $\lambda^{-n} P$, and hence when $B$ has the same center as $P$. The general case can be deduced fairly simply from this case and the doubling property of $\mu$.

Remark 2.2. The lemma holds under the weaker assumption that annuli in $\mathcal{S}$ are not empty, i.e, that $B(x, R) \backslash B(x, r), x \in \mathcal{S}$ and $0<r<R<\infty$, is never empty. See also [W, p. 269].

2.2. Whitney-type balls. In the proof of Theorem 1.2 we will need to make use of chains of balls that join a ball at one scale to another ball at a smaller scale. The proposition below will allow us to do this. A similar proposition appears in HaK2 in the case of metric spaces. We give a proof of the proposition because we no longer have a metric, and also to make explicit property iv) which will be important in the next section.

For a point $x \in \mathcal{S}$ and $0<\epsilon \leq(2 K)^{-1}$ the family of Whitney balls is defined by

$$
\mathcal{W}_{\epsilon}(x)=\{B(y, \epsilon d(y, x)): y \in \mathcal{S} \backslash\{x\}\} .
$$

Our choice of $\epsilon$ ensures that the following important properties hold:

i) For any $B \in \mathcal{W}_{\epsilon}(x)$ and any $z \in B$ we have $\frac{1}{2 K} \leq \frac{d(z, x)}{d\left(x_{B}, x\right)} \leq 2 K$. Observe that the radii are comparable to the distance to $x$; more specifically, $\frac{1}{2 K \epsilon} \leq$ $\frac{d(z, x)}{r_{B}} \leq \frac{2 K}{\epsilon}$.

ii) If $B_{1}$ and $B_{2}$ are two balls in $\mathcal{W}_{\epsilon}(x)$ whose intersection is not empty, then $\frac{1}{4 K^{2}} \leq \frac{r_{B_{1}}}{r_{B_{2}}} \leq 4 K^{2}$ and consequently $B_{2} \subseteq 10 K^{4} B_{1}$.

By a chain we mean a collection of sets $\left\{V_{j}\right\}, 1 \leq j \leq m$, such that $V_{j} \cap V_{k} \neq \emptyset$ if, and only if, $|j-k| \leq 1$. The $V_{j}$ are called the links of the chain. Two non-empty sets $A$ and $B$ are said to be linked by a family of sets $\mathcal{O}$ if there is a chain whose first link is $A$, whose last link is $B$, and whose remaining links (if any) lie in $\mathcal{O}$.

The following is a standard result from point set topology (Theorem 9, p.136 of $\mathrm{Ku}$ )

Lemma 2.3. Any two non-empty subsets of a connected space are linked by any open cover of the space.

Proposition 2.4. Suppose that $\mathcal{S}$ is connected and that $B(x, R)^{c}$ is not empty. Then for any $0<r<R$ there is a chain having the following properties:

i) All the links of the chain are elements of $\mathcal{W}_{\epsilon}(x)$ with centers in the annulus $A(R, r)=\{y: r \leq d(y, x) \leq R\}$.

ii) The first link has radius comparable to $\epsilon R$.

iii) The last link has non-empty intersection with $B(x, r)$ and has radius comparable to $\epsilon r$. 
iv) The number of links in the chain is at most $c \epsilon^{-D}(1+\log (R / r))$.

Proof. Set $U=\{y: d(y, x)>R\}$ (this may be empty). Then $U, B(x, r)$, and the elements of $\mathcal{W}_{\epsilon}(x)$ with centers in $A(R, r)$ form an open cover of $\mathcal{S}$. Apply the lemma using this open cover to the sets $B(x, R)^{c}$ and $B(x, r)$. We get a chain $\mathcal{C}_{0}$ whose first link is $B(x, R)^{c}$, whose last link is $B(x, r)$, and whose remaining links come from the open cover. In fact, a simple argument using the definition of a chain shows that the remaining links are neither $U$ nor $B(x, r)$. There are at least three links in the chain, because $r<R$. Removing the first and last links, we get a new chain $\mathcal{C}$ satisfying i). We will show that this is the chain we seek.

We will use the following observation, which is an easy consequence of property i) of $\mathcal{W}_{\epsilon}(x)$. For any ball $B \in \mathcal{W}_{\epsilon}(x)$ having non-empty intersection with $B(x, \lambda)$ and with $B(x, \lambda)^{c}$, we have

$$
\frac{\epsilon \lambda}{2 K} \leq r_{B} \leq 2 K \epsilon \lambda
$$

The second link of $\mathcal{C}_{0}$ intersects both the third link and the first link. It follows that the second link intersects both $B(x, R)^{c}$ and $B(x, R)$. However, the second link is in $\mathcal{W}_{\epsilon}(x)$ and so, by the inequality above, this link has radius comparable to $\epsilon R$. The second link of $\mathcal{C}_{0}$ is the first one of $\mathcal{C}$, so we have proven ii). Essentially the same argument proves iii).

Now, a simple argument, using $A(r, R) \subset \bigcup_{j=1}^{M} A\left(\frac{R}{2^{j}}, \frac{R}{2^{j-1}}\right)$ with $M \approx \log \frac{R}{r}$, shows that iv) follows if the number of links of $\mathcal{C}$ that intersect an annulus of the form $A\left(2^{-N+1}, 2^{-N}\right)$ is bounded by a constant times $\epsilon^{-D}$ (independently of $N$ ). Let $M_{N}$ be the number of links intersecting this annulus. Property i) of $\mathcal{W}_{\epsilon}(x)$ implies that any element of $\mathcal{W}_{\epsilon}(x)$ intersecting this annulus has radius comparable to $2^{-N} \epsilon$. This implies that all the relevant links lie in a ball $B_{N}=B\left(x, c 2^{-N}\right)$ and, by (16), that each one has measure at least $c_{1}^{-1} \epsilon^{D} \mu\left(B_{N}\right)$. The definition of a chain implies that any point of $\mathcal{S}$ can only lie in at most two links. Consequently, $M_{N} c_{1}^{-1} \epsilon^{D} \mu\left(B_{N}\right) \leq 2 \mu\left(B_{N}\right)$, and so $M_{N} \leq 2 c_{1} \epsilon^{-D}$.

\section{TRUDINGER'S TYPE INEQUALITY FOR CONNECTED SPACES}

The purpose of this section is to prove Theorem[1.2. We fix $\delta>0$ and a ball $B_{0}$. Recall that we are assuming that $f$ is a locally integrable function for which there exist constants $\tau \geq 1$ and $\|f\|_{a}>0$ such that for all balls $B$

$$
\frac{1}{\mu(B)} \int_{B}\left|f-f_{B}\right| d \mu \leq\|f\|_{a} a(\tau B),
$$

where $a$ is a functional satisfying the $T_{p}$ condition (Definition 1.1). The constants $K, D$, and $c_{\mu}$ will be referred to as the geometric data, and any constant that depends only on these will be called a geometric constant. Constants denoted by $c, c_{0}, \ldots$ will be geometric constants. All other constants will depend (at most) on the geometric data, $\tau, \delta^{-1},\|a\|, p$, and the doubling constants of $w$. We can, and do, make the assumption that $\|f\|_{a}=1$.

We will make constant use of the next lemma, often without referring to it. It holds for any functional $a$, not simply those satisfying the condition $T_{p}$.

Lemma 3.1. If $B$ and $P$ are balls with $P$ contained in $B$, then

$$
\left|f_{B}-f_{P}\right| \leq c_{\mu}\left(\frac{r(B)}{r(P)}\right)^{D} a(\tau B) .
$$


The lemma follows easily from (16) and (19). The next result shows that the polynomial control of the growth of the averages given in the lemma can be improved to logarithmic control once we assume that $a$ satisfies $T_{p}$. The key here is Proposition 2.4

Proposition 3.2. Let $B$ be a ball and $\lambda \leq 1$. Then

$$
\left|f_{B}-f_{\lambda B}\right| \leq C\left(1+\log \lambda^{-1}\right)^{1 / p^{\prime}} a(2 K \tau B) .
$$

Proof. Let $\lambda<1$; then, by replacing $B$ by a smaller multiple of itself, if necessary, and by using the previous lemma, we can assume that $B^{c}$ is not empty.

Set $\epsilon=(20 K)^{-5} \tau^{-1}$. Let $x$ be the centre of $B, R$ the radius of $B$, and $\mathcal{C}=$ $\left\{B_{i}\right\}_{i=1}^{N}$ the chain obtained by applying Proposition 2.4 with the $\epsilon$ just defined and $r=\lambda R$. One consequence of Proposition 2.4 i) is that all of the $B_{i}$ lie in $2 K B$. Part ii) of the same proposition says that the radius of $B_{1}$ is comparable to $R$, and so, by the previous lemma,

$$
\left|f_{B_{1}}-f_{B}\right| \leq C a(2 K \tau B) .
$$

In a similar manner, Proposition 2.4 iii) implies that $\left|f_{B_{N}}-f_{\lambda B}\right| \leq C a\left(c_{0} \tau \lambda B\right)$. When $\lambda \leq c_{0}^{-1}$ this yields

$$
\left|f_{B_{N}}-f_{\lambda B}\right| \leq C a(2 K \tau B) .
$$

If $\lambda \geq c_{0}^{-1}$, then the proposition follows from the lemma, so we only need to consider the case $\lambda \leq c_{0}^{-1}$. In this case both (21) and (20) hold, and as a result we have reduced the proposition to showing that

$$
\left|f_{B_{N}}-f_{B_{1}}\right| \leq C\left(1+\log \lambda^{-1}\right)^{1 / p^{\prime}} a(2 K \tau B) .
$$

Now define $\widehat{B}_{i}$ to be $10 K^{4} B_{i}$ and $\epsilon_{0}$ to be $(2 K)^{-1}$. All the $B_{i}$ are elements of $\mathcal{W}_{\epsilon}(x)$, and property ii) of $\mathcal{W}_{\epsilon}(x)$ guarantees that $B_{i+1}$ is contained in $\widehat{B}_{i}$. Consequently, $\left|f_{B_{i}}-f_{B_{i+1}}\right| \leq c a\left(\tau \widehat{B}_{i}\right)$, and

$$
\begin{gathered}
\left|f_{B_{N}}-f_{B_{1}}\right| \leq c \sum_{i=1}^{N-1} a\left(\tau \widehat{B}_{i}\right) \leq c\left(\sum_{i=1}^{N-1} a\left(\tau \widehat{B}_{i}\right)^{p}\right)^{1 / p} N^{1 / p^{\prime}} \\
\leq C\left(1+\log \lambda^{-1}\right)^{1 / p^{\prime}}\left(\sum_{i=1}^{N-1} a\left(\tau \widehat{B}_{i}\right)^{p}\right)^{1 / p}
\end{gathered}
$$

The last inequality follows from Proposition 2.4 iv). Our choice of $\epsilon$ ensures that the balls $\tau \widehat{B}_{i}$ all lie in $2 K B$ and that they belong to $\mathcal{W}_{\epsilon_{0}}(x)$.

We claim that the balls $\left\{\tau \widehat{B}_{i}\right\}$ can be divided into $C_{0}+1$ subfamilies of disjoint balls. Assuming this for the moment, we apply the condition $T_{p}$ to each of the subfamilies to obtain

$$
\left(\sum_{i} a\left(\tau \widehat{B}_{i}\right)^{p}\right)^{1 / p} \leq C a(2 K \tau B),
$$

and this gives the result we seek.

It remains to justify the claim. Let $\mathcal{F}$ denote the family of balls $\tau \widehat{B}_{i}$. If we can show that any element of $\mathcal{F}$ intersects at most $C_{0}$ other elements, then a simple counting argument proves the claim. Suppose that $F \in \mathcal{F}$ intersects $M$ other elements of $\mathcal{F}$. Denote these by $F_{1}, \ldots, F_{M}$. Let $z \in F_{i} \cap F$. As $\mathcal{F} \subseteq \mathcal{W}_{\epsilon_{0}}(x)$, both 
$F$ and $F_{i}$ have radii comparable to $r_{z}=d(z, x)$. This implies that all of the $F_{i}$ lie in $B\left(x, c r_{z}\right)$ and have radius comparable to this ball. However, the $F_{i}$ have finite overlap bounded by 2 (as they are the links of a chain), and therefore, using the doubling of $\mu, M$ is bounded by a constant $C_{0}$.

We next introduce a maximal operator associated to the functional $a$ :

$$
A(x)=\sup _{B: x \in B \subset \tau \widehat{B}_{0}}\left(\frac{a(B)}{w(B)^{1 / p}}\right)\left(\frac{w\left(\tau \widehat{B}_{0}\right)^{1 / p}}{a\left(\tau \widehat{B}_{0}\right)}\right) .
$$

We always have $A(x) \geq 1$. The next lemma shows that $A$ satisfies a $(p, p)$ weak type property, and it will play an important role in the proof of Theorem 1.2 .

\section{Lemma 3.3.}

$$
w\left(\left\{x \in \tau \widehat{B}_{0}: A(x)>\lambda\right\}\right) \leq \frac{C\|a\|^{p}}{\lambda^{p}} w\left(\tau \widehat{B}_{0}\right),
$$

and as a consequence for $0<s<p$ we have

$$
\left(\frac{1}{w\left(\tau \widehat{B}_{0}\right)} \int_{B_{0}} A(x)^{s} d w\right)^{1 / s} \leq C_{s}\|a\|
$$

Proof. The proof is by a standard covering lemma. If $A(x)>\lambda$, then for some ball $P \subset \tau \widehat{B}_{0}$ with $x \in P$ we have

$$
\lambda^{p} a\left(\tau \widehat{B}_{0}\right)^{p} \frac{w(P)}{w\left(\tau \widehat{B}_{0}\right)} \leq a(P)^{p} .
$$

Pick a Vitali type cover of $\left\{x \in \tau \widehat{B}_{0}: A(x)>\lambda\right\}$ by such balls $\left\{P_{i}\right\}$. Then the balls $\left\{P_{i}\right\}$ are pairwise disjoint sub-balls of $\tau \widehat{B}_{0}$, and $\left\{x \in \tau \widehat{B}_{0}: A(x)>\lambda\right\} \subset \bigcup_{i} c P_{i}$. Therefore, using $T_{p}$ and doubling of $w$,

$$
\begin{gathered}
w\left(\left\{x \in \tau \widehat{B}_{0}: A(x)>\lambda\right\}\right) \leq \sum_{i} w\left(c P_{i}\right) \leq C \sum_{i} w\left(P_{i}\right) \\
\leq \frac{C}{\lambda^{p}} \frac{w\left(\tau \widehat{B}_{0}\right)}{a\left(\tau \widehat{B}_{0}\right)^{p}} \sum_{i} a\left(P_{i}\right)^{p} \leq \frac{C\|a\|^{p}}{\lambda^{p}} w\left(\tau \widehat{B}_{0}\right) .
\end{gathered}
$$

The last statement follows from the general fact, known as Kolmogorov's inequality, that if $s<p$ then

$$
\left(\frac{1}{\nu(E)} \int_{E} g^{s} d \nu\right)^{1 / s} \leq C_{s}\|g\|_{p, \infty}(E, \nu)
$$

for any measurable set $E$ with finite measure on any arbitrary measure space $(X, \nu)$.

We will show that for $\omega$-a.e. $x \in B_{0}$

$$
\left|f(x)-f_{B_{0}}\right| \leq C a\left(\tau \widehat{B}_{0}\right)\left((\log A(x))^{1 / p^{\prime}}+1\right) .
$$

This pointwise estimate is the key. Once we have this, the theorem follows immediately. Indeed, let $s>0$. Then a simple manipulation of 23) yields

$$
\exp \left(s\left(\frac{\left|f(x)-f_{B_{0}}\right|}{C a\left(\tau \widehat{B}_{0}\right)}\right)^{p^{\prime}}\right) \leq \exp (s(1+\log A(x))) \leq C A(x)^{s}
$$


for $\omega$-a.e. $x \in B_{0}$. Averaging over $B_{0}$, we have

$$
\begin{aligned}
& \frac{1}{w\left(B_{0}\right)} \int_{B_{0}} \exp \left(s\left(\frac{\left|f(x)-f_{B_{0}}\right|}{C a\left(\tau \widehat{B}_{0}\right)}\right)^{p^{\prime}}\right) d w \\
& \quad \leq \frac{C}{w\left(B_{0}\right)} \int_{B_{0}} A(x)^{s} d w \leq \frac{C}{w\left(\tau \widehat{B}_{0}\right)} \int_{\tau \widehat{B}_{0}} A(x)^{s} d w .
\end{aligned}
$$

Choosing $s$ small enough, say $s=p / 2$, and appealing to Lemma 3.3 we find that the right-hand side is simply bounded by $C$, as required.

It remains to prove (23). By replacing $B_{0}$ by a smaller multiple of itself, if necessary, we can assume that $\tau \widehat{B}_{0} \neq \mathcal{S}$. Let $x \in B_{0}$ and set $\eta=\delta(2 K)^{-1}$. For $k=1,2, \ldots$ define $B_{k}$ to be $B\left(x, 2^{-k} \eta r\left(B_{0}\right)\right)$. Our choice of $\eta$ guarantees that $2 K \tau B_{k} \subset \tau \widehat{B}_{0}$. Both $B_{1}$ and $B_{0}$ lie in $\widehat{B}_{0}$; hence

$$
\left|f_{B_{1}}-f_{B_{0}}\right| \leq C a\left(\tau \widehat{B}_{0}\right) .
$$

This means that we can replace $f_{B_{0}}$ by $f_{B_{1}}$ in (23).

We are going to estimate $\left|f(x)-f_{B_{1}}\right|$ using some ideas going back to Hedberg's proof of the Sobolev embedding theorem by means of the Hardy-Littlewood maximal function. See $[\mathrm{AH}]$, Chapter 3, for example. By the Lebesgue differentiation theorem, we may assume that $\lim _{s \rightarrow 0} f_{B(x, s)}=f(x)$ for $\mu$-a.e., and hence for $\omega$-a.e., $x \in B_{0}$. Let $N=N(x)$ be a natural number to be chosen in a moment. Then,

$$
\left|f(x)-f_{B_{1}}\right| \leq\left|f_{B_{1}}-f_{B_{N}}\right|+\sum_{k=N}^{\infty}\left|f_{B_{k+1}}-f_{B_{k}}\right|=I+I I .
$$

Using Proposition 3.2 and the fact that $2 K \tau B_{1}$ is contained in $\tau \widehat{B}_{0}$, we find that

$$
I \leq C N^{1 / p^{\prime}} a\left(2 K \tau B_{1}\right) \leq C N^{1 / p^{\prime}} a\left(\tau \widehat{B}_{0}\right) .
$$

Now to the second term:

$$
\begin{gathered}
I I \leq \sum_{k=N+1}^{\infty} a\left(\tau B_{k}\right) \leq \sum_{k=N+1}^{\infty} \frac{a\left(\tau B_{k}\right)}{w\left(\tau B_{k}\right)^{1 / p}} w\left(\tau B_{k}\right)^{1 / p} \\
\leq A(x) \frac{a\left(\tau \widehat{B}_{0}\right)}{w\left(\tau \widehat{B}_{0}\right)^{1 / p}} \sum_{k=N+1}^{\infty} w\left(B_{k}\right)^{1 / p} .
\end{gathered}
$$

The balls $B_{k}$ all lie in $\tau \widehat{B}_{0}$ and $\tau \widehat{B}_{0}$ is not the whole space; therefore Proposition 2.1 implies that $w\left(B_{k}\right) \leq C \rho^{-k p} w\left(\tau \widehat{B}_{0}\right)$ for some constant $\rho>1$. Thus

$$
I I \leq C A(x) a\left(\tau \widehat{B}_{0}\right) \rho^{-N} .
$$

Combining our estimates for $I$ and $I I$, we have

$$
\left|f(x)-f_{B_{1}}\right| \leq C a\left(\tau \widehat{B}_{0}\right)\left(N^{1 / p^{\prime}}+A(x) \rho^{-N}\right) .
$$

Finally, choose $N$ to be the first integer strictly greater than $(\log A(x))(\log \rho)^{-1}$. Then

$$
\left|f(x)-f_{B_{1}}\right| \leq C a\left(\tau \widehat{B}_{0}\right)\left((\log A(x))^{1 / p^{\prime}}+1\right)
$$

and, as mentioned before, this yields (23). This completes the proof of Theorem 1.2 . 


\section{TRUdinger's inEQUALity is FALSE FOR NONCONNECTED SPACES}

Consider the subsets of $R^{d}$ given by $\mathcal{S}_{k}=B_{k} \cup\{0\}$, where $B_{k}$ is the closed ball centered at $\left(2^{-k}, 0, \cdots, 0\right)$ and of radius $2^{-k} / 10$. Each pair of the $\mathcal{S}_{k}$ have precisely one point in common, namely, the origin. Denote the Euclidean metric restricted to $\mathcal{S}_{k}$ by $\rho_{k}$. Now set $\mathcal{S}=\bigcup_{k=0}^{\infty} \mathcal{S}_{k}$. We can use the $\rho_{k}$ to define a metric on $\mathcal{S}$ by "passing" through the origin. Set

$$
\rho(a, b)= \begin{cases}\rho_{k}(a, b) & \text { if } a, b \in B_{k}, \\ \rho_{k}(a, 0)+\rho_{j}(0, b) & \text { if } a \in B_{k}, b \in B_{j}, \text { and } k \neq j .\end{cases}
$$

It is easy to see that $\rho$ is a metric and that it is equivalent to the usual Euclidean metric on $\mathcal{S}$. There is an explicit formula for $\rho$ :

$$
\rho(a, b)= \begin{cases}|b-a| & \text { if } a, b \in B_{k} \text { for some } k \\ |a|+|b| & \text { otherwise. }\end{cases}
$$

If we now equip $\mathcal{S}$ with this metric and Lebesgue measure, we get a space of homogeneous type. Furthermore, it is compact and $d$-regular. This last means that the measure of each ball is comparable to $r^{d}$.

Take $f$ to be the function $\sum_{k=0}^{\infty} k \chi_{B_{k}}$ and define a functional $a$ by

$$
a(B)=\delta_{0}(B)= \begin{cases}1 & \text { if } 0 \in B \\ 0 & \text { otherwise. }\end{cases}
$$

It is obvious that $a \in T_{p}$ for all $p$. Observe that $f$ is essentially $\log \frac{1}{|x|}$ restricted to $\mathcal{S}$. Thus, the function is of exponential type and no better; in particular, $f \notin \exp L^{p}$ for any $p>1$. If we can show that

$$
\frac{1}{\mu(B)} \int_{B}\left|f-f_{B}\right| d \mu \leq C a(B)
$$

for every ball $B$, then we will be done.

There are two cases to consider: $0 \in B$ and $0 \notin B$. In the first case (24) holds because $f$ is in BMO. Suppose now that $0 \notin B$. The nature of the metric $\rho$ now implies that $B$ intersects only one of the balls $B_{k}$. This means that $f$ is constant on $B$, and so (24) holds again.

\section{Proof of the John-Nirenberg type inequality FOR A GENERAL SPACE OF HOMOGENEOUS TYPE}

In this section we prove Theorem 1.4. The proof is an adaptation to this context of one of the proofs of the John-Nirenberg theorem for $\mathbb{R}^{n}$. The idea dates back to Calderón, and a related proof can be found in [Jo]. We mention that [Buc] and [MMNO] also contain proofs of the John-Nirenberg theorem. However, our proof is more general, and shows the relationship with our generalized Trudinger's inequality and in particular with the $T_{p}$ condition.

We fix $\delta>0$ and assume that $f, a$, and $w$ are as in statement of the theorem. Recall that for a given ball $B, \widehat{B}$ denotes the ball $(1+\delta) K B$. As in the previous sections, constants denoted by $c, c_{0}, \ldots$ will be geometric constants. All other constants will depend (at most) on the geometric data, $\tau, \delta^{-1},\|a\|$, and the $A_{\infty}$ constants of $w$. 
It is clear that we can renormalize $f$ so that $\|f\|_{a}=1$. Thus our initial assumption is

$$
\frac{1}{\mu(B)} \int_{B}\left|f-f_{B}\right| d \mu \leq a(\tau B)
$$

for every ball $B$. Now, it will be convenient to redefine $a$. Set $\tilde{a}(B)$ to be the supremum of $a(P)$ over all balls $P$ contained in $B$. Then

$$
a(B) \leq \tilde{a}(B) \leq\|a\| a(B) .
$$

(25) holds with $a$ replaced by $\tilde{a}$, and $\tilde{a}$ satisfies $T_{\infty}$ with constant 1 , i.e., $\tilde{a}$ is increasing. We shall assume from now on that $a$ is increasing.

Define, for each ball $B$ and $t>0$,

$$
E(B, t)=\left\{x \in B: \frac{\left|f(x)-f_{B}\right|}{a(\tau \widehat{B})}>t\right\} \text { and } \varphi(t)=\sup _{B} \frac{w(E(B, t))}{w(B)} .
$$

We have to prove that for any ball $B$

$$
\left\|f-f_{B}\right\|_{\exp L(B, w)} \leq C a(\tau \widehat{B}) .
$$

This is equivalent to proving that there are positive constants $C_{0}$ and $C_{1}$ for which

$$
\varphi(t) \leq C_{1} e^{-t / C_{0}}
$$

for all $t>0$. Note that this inequality holds automatically when $t$ is not too large, as $\varphi(t)$ is always bounded by 1 . If we can show that

$$
\frac{w(E(B, t))}{w(B)} \leq \frac{1}{2} \varphi\left(t-t_{0}\right)
$$

for any $B$ and for all $t>t_{0}$, where $t_{0}$ is a positive constant independent of $B_{0}$, then iteration of this inequality yields (27).

Fix an arbitrary ball $B_{0}$. Observe that we may assume that $f_{B_{0}}=0$. We now follow the line of argument considered in $[\mathrm{MP}]$. Associated to the fixed ball $B_{0}$ we define the following basis:

$$
\mathcal{B}=\left\{B: x_{B} \in B_{0} \text { and } r_{B} \leq \delta r_{B_{0}}\right\} .
$$

This family has the following properties:

$$
\begin{gathered}
B \in \mathcal{B} \Rightarrow B \subseteq \widehat{B}_{0}, \\
B \in \mathcal{B} \Rightarrow \tau B \subseteq \tau \widehat{B}_{0} .
\end{gathered}
$$

The maximal function associated to $\mathcal{B}$ of a locally integrable function $g$ is

$$
M_{\mathcal{B}} g(x)=\sup _{B: x \in B \in \mathcal{B}} \frac{1}{\mu(B)} \int_{B}|g| d \mu,
$$

where we understand that the supremum is zero if $x$ is not contained in any element of $\mathcal{B}$. This function is zero outside $\widehat{B}_{0}$. The Lebesgue differentiation theorem and the definition of the basis $\mathcal{B}$ imply that $|g(x)| \leq M_{\mathcal{B}} g(x)$ for $\mu$-a.e., and hence for $\omega$-a.e., $x \in B_{0}$. For $t>0$ set

$$
\Omega_{t}=\left\{x \in \mathcal{S}: M_{\mathcal{B}} f(x)>\operatorname{ta}\left(\tau \widehat{B}_{0}\right)\right\} .
$$

This set is contained in $\widehat{B}_{0}$. For notational convenience we introduce

$$
\operatorname{Av}(B)=\frac{1}{\mu(B)} \int_{B}|f| d \mu .
$$


Notice that since $f_{B_{0}}=0$ we have that $\operatorname{Av}\left(\widehat{B}_{0}\right) \leq C a\left(\tau \widehat{B}_{0}\right)$ and that $E\left(B_{0}, t\right) \subseteq \Omega_{t}$.

The following lemma, although easy, is nevertheless important.

Lemma 5.1. Let $B \in \mathcal{B}$ be such that $\operatorname{Av}(B)>t a\left(\tau \widehat{B}_{0}\right)$. Then

$$
r(B) \leq c_{0}\left(\frac{1}{t}\right)^{1 / D} r\left(B_{0}\right) .
$$

Indeed, since $B \subset \widehat{B}_{0}$ we have

$$
\begin{aligned}
\operatorname{ta}\left(\tau \widehat{B}_{0}\right)<\operatorname{Av}(B) \leq \frac{\mu\left(\widehat{B}_{0}\right)}{\mu(B)} \operatorname{Av}\left(\widehat{B}_{0}\right) & \leq c_{\mu}\left(\frac{r\left(\widehat{B}_{0}\right)}{r(B)}\right)^{D} \operatorname{Av}\left(\widehat{B}_{0}\right) \\
& \leq c_{0}^{D}\left(\frac{r\left(B_{0}\right)}{r(B)}\right)^{D} a\left(\tau \widehat{B}_{0}\right),
\end{aligned}
$$

and the lemma follows.

Set $M=2(1+\delta) K^{2}(2 K+1)$ and let $t_{0}>\left(\frac{c_{0} M}{\delta}\right)^{D}$ be a constant to be specified more precisely later on. Observe that for $t>t_{0}$ we have by Lemma 5.1 that

$$
r(B) \leq \frac{\delta}{M} r\left(B_{0}\right)
$$

whenever $B \in \mathcal{B}$ with $\operatorname{Av}(B)>\operatorname{ta}\left(\tau \widehat{B}_{0}\right)$.

We need the following Calderon-Zygmund or stopping-time type lemma.

Lemma 5.2. Suppose that $\Omega_{t}$ is not empty. Then there exists a countable family $\left\{B_{i}\right\}$ of pairwise disjoint balls in $\mathcal{B}$ such that:

i) $\bigcup_{i} B_{i} \subseteq \Omega_{t} \subseteq \bigcup_{i} B_{i}^{*}$, where $B^{*}=K(2 K+1) B$.

ii) For each $i, r\left(B_{i}\right) \leq \frac{\delta}{M} r\left(B_{0}\right)$.

iii) $\operatorname{Av}\left(B_{i}\right)>\operatorname{ta}\left(\tau \widehat{B}_{0}\right)$ for all $i$.

iv) If $\sigma B_{i} \in \mathcal{B}$ and $\sigma \geq 2$, then $\operatorname{Av}\left(\sigma B_{i}\right) \leq \operatorname{ta}\left(\tau \widehat{B}_{0}\right)$.

Proof. For $x \in \Omega_{t}$ there exists a ball $B^{\prime} \in \mathcal{B}$ with $x \in B^{\prime}$ such that $\operatorname{Av}\left(B^{\prime}\right)>$ ta $\left(\tau \widehat{B}_{0}\right)$. Let $R=R_{x}$ be defined by

$$
R=\sup \left\{r(B): B \in \mathcal{B}, B \text { is a multiple of } B^{\prime} \text { and } \operatorname{Av}(B)>t a\left(\tau \widehat{B}_{0}\right)\right\} .
$$

Lemma 5.1 and the range of $t$ imply that $R \leq \frac{\delta}{M} r\left(B_{0}\right)$. There exists a ball $B_{x}$ which is a multiple of $B^{\prime}$, whose radius satisfies $R / 2<r\left(B_{x}\right) \leq R$, and for which $\operatorname{ta}\left(\tau \widehat{B}_{0}\right)<\operatorname{Av}\left(B_{x}\right)$. This ball satisfies ii), iii), and iv). Part iii) implies that $\bigcup_{x \in \Omega_{t}} B_{x} \subseteq \Omega_{t}$, while we obviously have $\Omega_{t} \subseteq \bigcup_{x \in \Omega_{t}} B_{x}$. Thus these two inclusions are actually equalities. Picking a Vitali type subcover of $\left\{B_{x}\right\}_{x \in \Omega_{t}}$ gives us a family of pairwise disjoint balls $\left\{B_{i}\right\} \subset\left\{B_{x}\right\}_{x \in \Omega_{t}}$ satisfying i).

Let $E(t)=E\left(B_{0}, t\right)$. Since $t>t_{0}$ and $E\left(t_{0}\right) \subseteq \Omega_{t_{0}}$, we can apply the lemma to $\Omega_{t_{0}}$ to get

$$
E_{t}=E_{t_{0}} \cap E_{t} \subseteq \Omega_{t_{0}} \cap E_{t} \subseteq \bigcup_{i} B_{i}^{*} \cap E_{t}
$$


Now, for $x \in B_{i}^{*} \cap E_{t}$ we have

$$
\begin{gathered}
\operatorname{ta}\left(\tau \widehat{B}_{0}\right)<|f(x)| \leq\left|f(x)-f_{2 B_{i}^{*}}\right|+\left|f_{2 B_{i}^{*}}\right| \\
\leq\left|f(x)-f_{2 B_{i}^{*}}\right|+\operatorname{Av}\left(2 B_{i}^{*}\right) \leq\left|f(x)-f_{2 B_{i}^{*}}\right|+t_{0} a\left(\tau \widehat{B}_{0}\right) .
\end{gathered}
$$

In the last inequality we have used iv) in Lemma 5.2 and the fact that $2 B_{i}^{*} \in \mathcal{B}$ by our choice of $t_{0}$. Consequently,

$$
\left(t-t_{0}\right) a\left(\tau \widehat{B}_{0}\right) \leq\left|f(x)-f_{2 B_{i}^{*}}\right| .
$$

Now we claim that $\widehat{2 B_{i}^{*}}$ belongs to $\mathcal{B}$. Recall that the ball $B$ belongs to the basis $\mathcal{B}$ if its center is in $B_{0}$ and its radius is bounded by $\delta$ times the radius of $B_{0}$. It is clear that the center of $\widehat{2 B_{i}^{*}}$ is in $B_{0}$, since $B_{i}$ belongs to $\mathcal{B}$. Now observe that the radius of $\widehat{2 B_{i}^{*}}$ equals $\operatorname{Mr}\left(B_{i}\right) \leq \delta r\left(B_{0}\right)$ by property ii) of Lemma 5.2 This proves the claim. We continue by using property (31) to deduce that $\tau \widehat{2 B_{i}^{*}} \subseteq \tau \widehat{B}_{0}$ and the fact that $a$ is an increasing functional to obtain

$$
\left(t-t_{0}\right) a\left(\tau \widehat{2 B_{i}^{*}}\right) \leq\left|f(x)-f_{2 B_{i}^{*}}\right| .
$$

Consequently, $B_{i}^{*} \cap E_{t} \subset E\left(2 B_{i}^{*}, t-t_{0}\right)$. Thus

$$
\begin{aligned}
w\left(E_{t}\right) & \leq \sum_{i} w\left(2 B_{i}^{*}\right) \frac{w\left(E\left(2 B_{i}^{*}, t-t_{0}\right)\right)}{w\left(2 B_{i}^{*}\right)} \leq \varphi\left(t-t_{0}\right) \sum_{i} w\left(2 B_{i}^{*}\right) \\
& \leq C \varphi\left(t-t_{0}\right) \sum_{i} w\left(B_{i}\right)=C \varphi\left(t-t_{0}\right) w\left(\bigcup_{i} B_{i}\right)
\end{aligned}
$$

In the last equality we used that the balls $\left\{B_{i}\right\}$ are disjoint. The balls $B_{i}$ all lie in $\widehat{B}_{0}$; therefore $w$ being $A_{\infty}$ with respect to $\mu$ implies that there is a positive constant $\alpha$ for which

$$
w\left(\bigcup_{i} B_{i}\right) \leq C\left(\frac{\mu\left(\bigcup_{i} B_{i}\right)}{\mu\left(\widehat{B}_{0}\right)}\right)^{\alpha} w\left(\widehat{B}_{0}\right) .
$$

We now use iii) of Lemma 5.2 and the observation that $\operatorname{Av}\left(\widehat{B}_{0}\right) \leq a\left(\tau \widehat{B}_{0}\right)$ to obtain

$$
\mu\left(\bigcup_{i} B_{i}\right)=\sum_{i} \mu\left(B_{i}\right) \leq \sum_{i} \frac{1}{a\left(\tau \widehat{B}_{0}\right) t_{0}} \int_{B_{i}}|f| d \mu \leq \frac{1}{a\left(\tau \widehat{B}_{0}\right) t_{0}} \int_{\widehat{B}_{0}}|f| d \mu \leq \frac{\mu\left(\widehat{B}_{0}\right)}{t_{0}} .
$$

It follows that

$$
w\left(E_{t}\right) \leq C \varphi\left(t-t_{0}\right) \frac{w\left(\widehat{B}_{0}\right)}{t_{0}^{\alpha}} \leq C_{4} \frac{w\left(B_{0}\right)}{t_{0}^{\alpha}} \varphi\left(t-t_{0}\right)
$$

Choosing $t_{0}=\max \left\{\left(2 C_{4}\right)^{1 / \alpha},\left(\frac{c_{0} M}{\delta}\right)^{D}\right\}$ yields (28), and so completes the proof of the theorem. 


\section{REFERENCES}

[Ad] D.R. Adams, A sharp inequality of Moser for higher order derivatives, Annals of Mathematics 128 (1988), 385-398. MR 89i:46034

[AH] D.R. Adams and L.I. Hedberg, Function spaces and potential theory, Grundlehren Math. Wiss., vol. 314, Springer Verlag, (1996). MR 97j:46024

[B] Bojarski, B., Remarks on Sobolev imbedding inequalities, Lecture Notes in Math. 1351, Springer, 1988, 52-68. MR 90b:46068

[Bo] Boman, J. $L^{p}$-estimates for very strongly elliptic systems, Department of Mathematics, University of Stockholm, Sweden (1982), Report no. 29.

[Buc] S. Buckley, Inequalities of John-Nirenberg type in doubling spaces, J. d'Analyse Math. 79 (1999), 215-240. MR 2001f:46059

$[\mathrm{Bu}] \quad$ P. Buser, A note on the isoperimetric constant, Ann. Scient. Ec. Norm. Sup. 15 (1982), 213-230. MR 84e:58076

[CW] R. Coifman and G. Weiss, Analyse harmonique non-commutative sur certains espaces homogènes, Lect. Notes Math. 242, Springer Verlag, (1971). MR 58:17690

[FP] C. Fefferman and D. H. Phong, Subelliptic eigenvalue problems, Conf. on Harmonic Anal. in Honor of A. Zygmund (Chicago, 1981), Wadsworth, Belmont, CA, 1983, pp. 590-606. MR 86c:35112

[Fr] B. Franchi, Inégalités de Sobolev pour des champs de vecteurs lipschitziens, C. R. Acad. Sci. Paris Sér. I Math. 311 (1990), 329-332. MR 91k:46028

[FGW] Franchi, B., Gutiérrez, C. E., Wheeden, R. L., Weighted Sobolev-Poincaré inequalities for Grushin type operators, Comm. Partial Differential Equations 19 (1994), 523-604. MR 96h:26019

[FPW] B. Franchi, C. Pérez and R. L. Wheeden, Self-improving properties of John-Nirenberg and Poincaré inequalities on spaces of homogeneous type, J. Funct. Anal. 153 (1998), 108-146. MR 99d:42042

[GN] N. Garofalo and D. Nhieu, Isoperimetric and Sobolev inequalities for CarnotCarathédory spaces and the existence of minimal surfaces, Communications in Pure and Applied Mathematics, 69 (1996), 1081-1144. MR 97i:58032

[GT] D. Gilbarg and N.S. Trudinger, Elliptic partial differential equations of second order, 2nd ed., Grundlehren Math. Wiss., vol. 69, Springer-Verlag, (1983).

[HaK1] P. Hajłasz and P. Koskela, Sobolev meets Poincaré, C. R. Acad. Sci. Paris Sér. I Math. 320 (1995), 1211-1215. MR 96f:46062

[HaK2] P. Hajłasz and P. Koskela, Sobolev met Poincaré, Mem. Amer. Math. Soc. 145 (2000), No. 688. MR 2000j: 46063

[Je] D. Jerison, The Poincaré inequality for vector fields satisfying Hörmander's condition, Duke Math. J. 53 (1986), 503-523. MR 87i:35027

[Jo] J. L. Journé, Calderón-Zygmund operators, pseudo-differential operators and the Cauchy integral of Calderón, Lecture Notes in Math. 994 (1983), Springer Verlag. MR 85i:42021

$[\mathrm{Ku}] \quad$ K. Kuratowski, Topology, Vol II, Academic Press, (1968). MR 41:4467

[L1] G. Lu, Embedding theorems on Campanato-Morrey spaces for vector fields of Hörmander, Approx. Theory Appl. (N.S.) (1998), no. 1, 69-80. MR 94h:46053

[L2] G. Lu, The sharp Poincaré inequality for free vector fields: An endpoint result, Revista Mat. Iberoamericana 10 (1994), 453-466. MR 96g:26023

[MP] P. MacManus and C. Pérez, Generalized Poincaré inequalities: Sharp self-improving properties, International Math. Research Notices 1998, no. 2, 101-116. MR 99k:42045

[MS] R. Macias and C. Segovia, Lipschitz functions on spaces of homogeneous type, Adv. in Math. 33 (1979), 257-270. MR 81c:32017a

[MMNO] J. Mateu, P. Mattila, A. Nicolau and J. Orobitg, BMO for nondoubling measures, Duke Math. J. 102 (2000), 533-565. MR 2001e:26019]

[Mo] J. Moser, A sharp form of an inequality of N. Trudinger, Indiana J. of Math. 20 (1971), 1077-1092. MR 46:662

[NSW] A. Nagel, E. M. Stein and S. Wainger, Balls and metrics defined by vector fields, I: Basic properties, Acta Math. 155 (1985), 103-147. MR 86k:46049

[SCa] A. Sánchez-Calle, Fundamental solutions and geometry of the sums of squares of vector fields, Invent. Math. 78 (1984), 142-160. MR 86e:58078 
[SCo] L. Saloff-Coste, Uniformly elliptic operators on Riemannian manifolds, J. Diff. Geometry 36 (1992), 417-450. MR 93m:58122

[SW] E. T. Sawyer and R. L. Wheeden, Weighted inequalities for fractional integrals on euclidean and homogeneous spaces, Amer. J. Math. 114 (1992), 813-874. MR 94i:42024

[ST] J.-O. Strömberg and A. Torchinsky, Weighted Hardy spaces, Lec. Notes in Math. Springer-Verlag 1381 (1989). MR 90j:42053

[Y] V. I. Yudovich, Some estimates connected with integral operators and with solutions of elliptic equations, Dokl. Akad. Nauk SSRR 138 (1961), 805-808 (Rusian); English transl., Soviet Math. Dokl. 2 (1961), 746-749. MR 25:4236

[W] R. L. Wheeden, A characterization of some weighted norm inequalities for the fractional maximal function, Studia Math. 107 (1993), 251-272. MR 94m:42044

Department of Mathematics, National University of Ireland, Maynooth, Co. KilDARE, IRELAND

Current address: Phillips Exeter Academy, 20 Main St., Exeter, New Hampshire 03833

E-mail address: pmacmanus@exeter.edu

Departamento de Análisis Matemático, Facultad de Matemáticas, Universidad de Sevilla, 41080 Sevilla, Spain

E-mail address: carlosperez@us.es 